\title{
Klebsiella pneumoniae infection secondary to bovine viral diarrhea in two prematurely born calves
}

\author{
Kyunghyun Lee ${ }^{1}$, Ha-Young Kim ${ }^{1}$, Eun-Jin Choi ${ }^{1}$, Kyoung-Ki Lee ${ }^{1}$, ByungJae So ${ }^{1}$, Ji-Youl Jung ${ }^{1,2, *}$ \\ ${ }^{1}$ Animal Disease Diagnostic Division, Animal and Plant Quarantine Agency, Gimcheon 39660, Korea \\ ${ }^{2}$ College of Veterinary Medicine and Veterinary Medical Research Institute, Jeju National University, Jeju 63243, Korea
}

\begin{abstract}
This paper describes the development of neurological signs of two prematurely born calves four days after birth. The pathological examination results indicated fibrinopurulent polyserositis, including meningoencephalitis with suppurative bronchopneumonia. Bovine viral diarrhea virus subtype $2 \mathrm{a}$ was detected in most of the internal organs, and the bacterial colonies cultured from the samples were identified as Klebsiella $(K$.) pneumoniae. Molecular analysis via multilocus sequence typing identified a different $K$. pneumoniae isolate in each calf-type 14 in calf A and type 65 in calf B. This is the first report identifying $K$. pneumoniae sequence types 14 and 65 in cattle.
\end{abstract}

Keywords: bovine viral diarrhea, calf, Klebsiella pneumoniae, Korea, polyserositis

*Corresponding author

Ji-Youl Jung

Veterinary Medical Research Institute, College of Veterinary Medicine, Jeju National

University, 102 Jejudaehak-ro, Jeju 63243, Korea

Tel: $+82-64-754-3364$

Fax: +82-64-702-9920

E-mail: jungjy1982@jejunu.ac.kr

Conflict of Interest

The authors have no conflicts of interest to declare.

ORCID:

Kyunghyun Lee

https://orcid.org/0000-0002-3113-2781

Ha Young Kim

http://orcid.org/0000-0002-1332-3832

Eun-Jin Choi

https://orcid.org/0000-0001-9690-7501

Kyoung-Ki Lee

https://orcid.org/0000-0002-6832-6264

ByungJae So

https://orcid.org/0000-0002-6125-6873

Ji-Youl Jung

https://orcid.org/0000-0002-5938-2029

Received: August 3, 2020

Revised: August 14, 2020

Accepted: August 19, 2020
Bovine viral diarrhea virus (BVDV) is a member of the Flaviviridae family that belongs to the genus Pestivirus [1]. The BVDV includes three species, BVDV-1, BVDV-2, and BVDV-3 [1]. Although BVDV infects several species of animals, diseases associated with BVDV have been recorded in most countries, where cattle are raised, and it causes significant economic losses via reduced reproductive capacity and the immunosuppression of herds [2]. A BVDV infection causes a wide variety of clinical symptoms in cattle, including abortion, congenital defects, fetal mummification, persistent infection, diarrhea, sudden death, and necrosis of the squamous epithelium in the oral cavity, esophagus, rumen, omasum, and skin [3].

Klebsiella (K.) pneumoniae is a gram-negative, aerobic, nonmotile bacillus of the family Enterobacteriaceae [4]. The bacterium exists widely in surface water, sewage, soil, plants, and the mucosal surfaces of mammals. Neonatal infections and sepsis occur most frequently in calves that experience the failure of passive transfer. K. pneumoniae is a common bacterial pathogen associated with septicemia in calves [5]. Despite several case reports documenting BVDV infections of cattle in Korea, reports of BVDV co-infections with $K$. pnuemoniae are lacking. This paper describes $K$. pneumoniae infection secondary to BVDV in two prematurely born calves.

In July 2017, a live calf was born prematurely on a farm with 184 Korean cattle. The cows at this farm were not vaccinated against the BVDV. Over two weeks, eight heifers in their last month of gestation aborted or gave birth to calves approximately two-four weeks early. No other observed clinical signs, besides abortion or premature birth, were observed in the heifers. Of these, five premature live calves appeared undersized for their age. Two of them were weak and exhibited neurological signs, such as lethargy and an inability to stand. Despite symptomatic treatment, their conditions did not improve, and they died four days after birth. Both calves were supplied to the Animal and Plant Quarantine Agency in Korea to determine the cause of death.

In calf $A$, yellowish fibrinous material was attached to the serosa of most internal organs, and a moderately turbid yellowish fluid was observed in the pleural and peritoneal cavity. Purple-red consolidation of the lungs was also noted (Fig. 1A). In calf B, a small amount of yellowish turbid ascites was observed. The loss of definition of gyri, meningeal cloudiness, and yellowish 
material in the brain sulci of both calves was observed (Fig. 1B).

Necropsies were performed, and the representative tissues were fixed in $10 \%$ neutral-buffered formalin and embedded in paraffin wax, after which $4 \mu \mathrm{m}$-thick sections were stained with hematoxylin and eosin, and gram-stained for the optical microscopy examination. A rabbit polyclonal anti-K. pneumoniae antibody (Abcam, ATCC\#43816, ab20947, UK) was used for immunohistochemical analysis. Staining was per- formed using a fully automated system (NexES IHC instrument; Ventana Medical System, USA) and a 3,3'-diaminobenzidine tetrahydrochloride detection system.

Histopathologically, fibrinopurulent serositis was observed in the pleural and peritoneal visceral organs, and moderate to severe suppurative bronchopneumonia (Fig. 2A) with interlobular edema was evident in the lungs of calf $\mathrm{A}$. In the bronchiolar and alveolar lumen, numerous neutrophils and macrophages were noted (Fig. 2A, inset). Severe diffuse
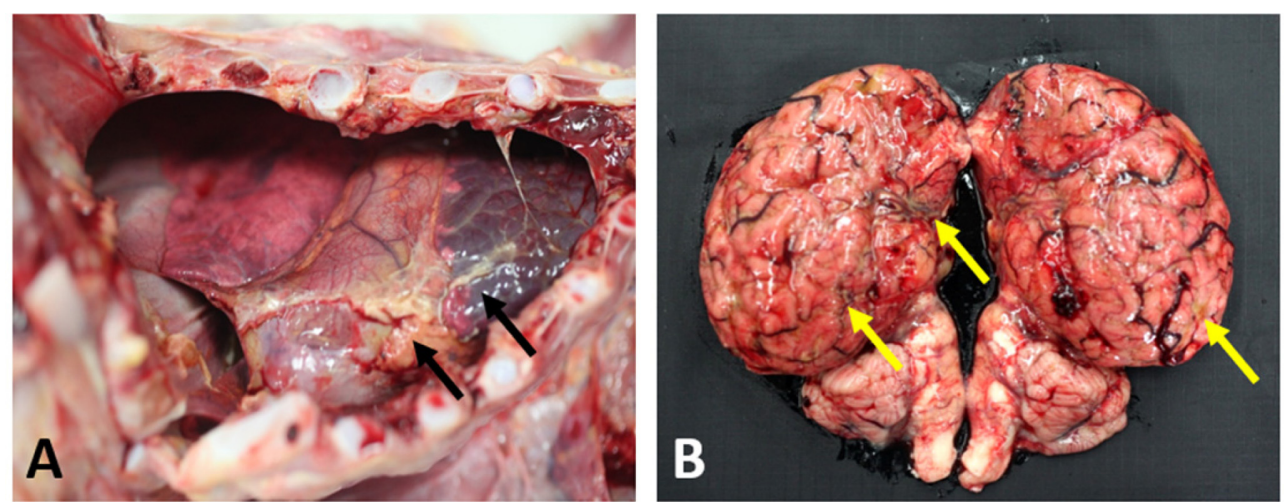

Fig. 1. Gross findings. (A) Calf A. Pulmonary consolidation and yellowish material in the pleura (black arrows) were observed. (B) Calf B. Loss of definition of the gyri, meningeal cloudiness, and yellowish material in the brain sulci (yellow arrows).
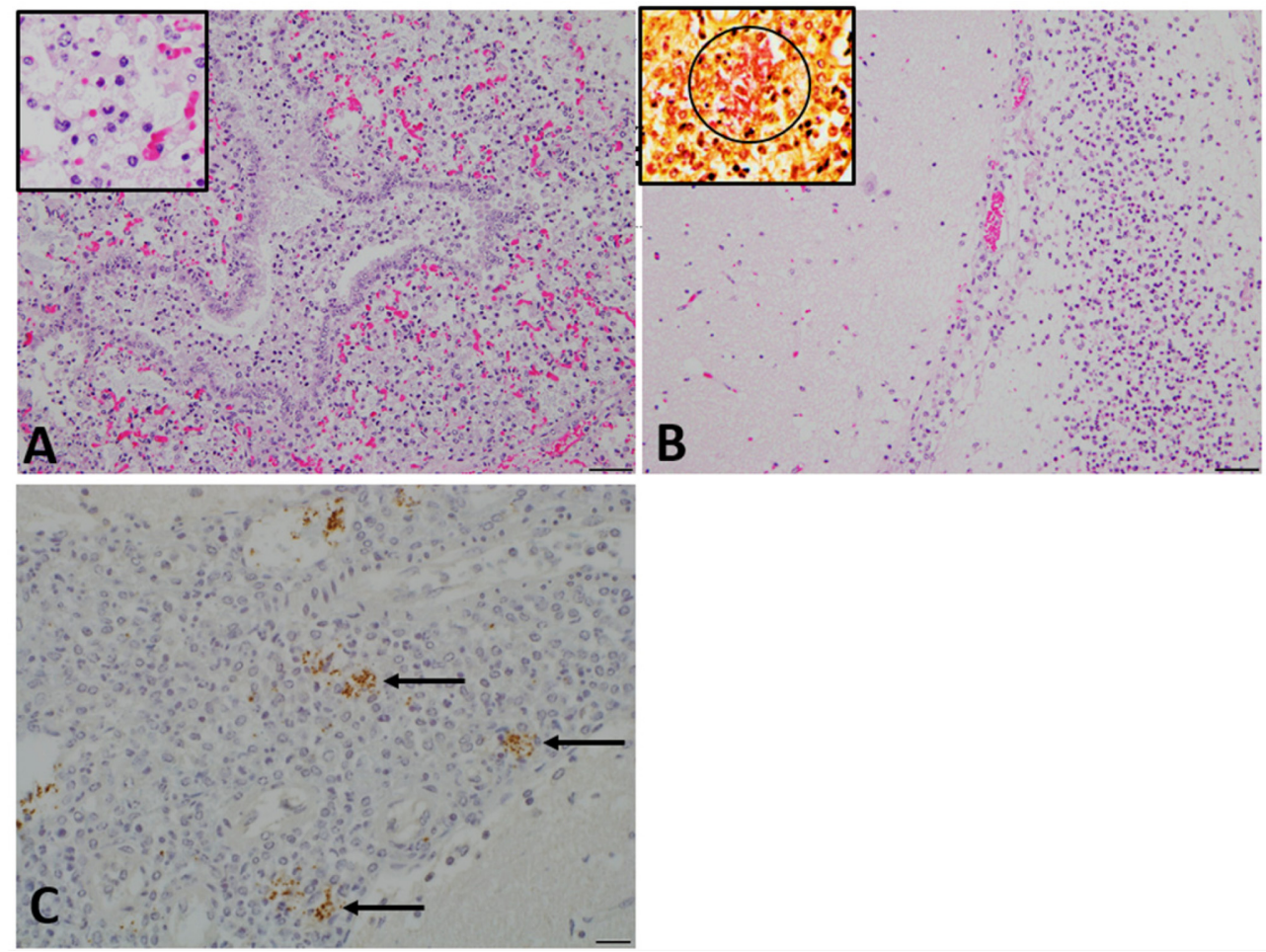

Fig. 2. Histopathological findings in two calves. (A) Lungs of calf A. Moderated suppurative bronchopneumonia. $\mathrm{H} \& \mathrm{E}, \mathrm{bar}=50 \mu \mathrm{m}$. Note the presence of neutrophils and macrophages in the alveoli (inset). (B) Brain of calf $\mathrm{B}$. Severe diffuse suppurative meningitis with numerous neutrophils. $\mathrm{H} \& \mathrm{E}, \mathrm{bar}=50 \mu \mathrm{m}$. Note the Gram- negative bacteria within the lesion (inset, circle). (C) Brain of calf B. Numerous bacilli (arrows) within the lesion react with an antibody against Klebsiella pneumoniae. IHC, bar $=20 \mu \mathrm{m}$. H \& E, hematoxylin and eosin. 
meningoencephalitis characterized by the infiltration of neutrophils and lymphocytes with numerous bacilli was observed in both calves (Fig. 2B). Numerous gram-negative bacilli were detected in the meninges (Fig. 2B, insert) and bronchopneumonia lesions. These bacilli stained positively with the aforementioned antibody against $K$. pneumoniae (Fig. 2C).

To determine the cause of the premature births and inflammation in the calves, a reverse transcription-PCR assay was performed using a kit (Lilif, BVD RT-PCR kit, Cat No. 99-43830) to identify BVDV RNA in the lungs, heart, liver, spleen, kidney, and brain tissues. All tissue samples were positive for the BVDV, and BVDV-2a was identified based on phylogenetic analysis of the 5' untranslated region [6]. The sera from six heifers that had affected calves were tested via the BVDV Ab enzyme-linked immunosorbent assay kit (IDEXX, Cat 99-43830). All the samples contained antibodies against the BVDV.

Umbilical cords, pleural and peritoneal effusion, lungs, and brain were harvested from both calves for bacterial culture. The samples were cultured on 5\% sheep blood agar (Asan Pharm., Korea) at $37^{\circ} \mathrm{C}$, and $K$. pneumoniae was isolated from all the samples. To investigate the detailed characteristics of six K. pneumoniae isolates, multilocus sequence typing (MLST) was performed using seven housekeeping genes (gapA, infB, $m d h$, pgi, phoE, rpoB, and tonB) [7]. The MLST results were compared with the international $K$. pneumoniae MLST database (http://bigsdb.web.pasteur.fr/klebsiella/klebsiella.html). The isolates from calf A were identified as ST14, and those from calf B were identified as ST65 (Table 1).

In Korea, BVDV causes considerable economic loss. Persistently infected calves can contribute to the spread of BVDV to other members of the herd [1]. The Korean government and cattle breeders are currently very interested in reducing the occurrence of the BVDV. Accordingly, improving herd immunity via vaccination and identifying and removing persistently infected animals from herds are believed to be effective preventive measures. At the farm where the two calves described in the current report were born, none of the heifers were vaccinated. Therefore, their susceptibility to infection was presumably relatively high. The serology results suggested that a BVDV infection may have caused the aforementioned abortions and premature births at this farm.

In most countries, the prevalence of BVDV-1 is higher than that of BVDV-2 [8]. To date, eight BVDV subtypes (1a-1d, 1m-1o, and 2a) have been identified in Korea [1]. The reports of previously assessed samples suggest that while numerous subtypes are present, subtypes BVDV-1b and BVDV-2a are predominant and widespread in Korea. Subtype BVDV-2a causes considerable economic loss to the Korean cattle industry- primarily through reproductive losses $[1,8]$. Subtype BVDV-2a was isolated from both the prematurely born calves described in the current report.

Klebsiella sp. can cause mastitis, leading to significant eco-
Table 1. Multilocus sequence typing data for Klebsiella pneumoniae isolates from calves

\begin{tabular}{clc}
\hline \hline Calf & Allelic profile & $\mathrm{ST}^{*}$ \\
\hline A & $1-6-1-1-1-1-1$ & 14 \\
B & $2-1-2-1-10-4-13$ & 65 \\
\hline
\end{tabular}

*Allelic profile, gapA-infB-mdh-pgi-phoE-rpoB-tonB; ${ }^{\dagger}$ ST, sequence type.

nomic losses on dairy farms. Many reports suggest that $K$. pneumoniae is one of the main causative agents of bovine mastitis $[9,10]$. Like most coliforms, $K$. pneumoniae is a commensal of the intestinal tracts of animals. Accordingly, environments with fecal contamination are often the source of perinatal infections in young ruminants. There is very little information available on $K$. pneumoniae isolated from septicemic cattle. Although the source of a $K$. pneumoniae infection in these two cases was not determined, the infection is believed to have been transmitted through the environment. Moreover, the primary sites of infection are believed to have been the umbilical cords, followed by hematogenous transmission to various other tissues. The calves exhibited neurological signs because of bacterial infection in the brain. Antibacterial therapy is used widely for the treatment of $K$. pneumoniae infections, but it will only have maximal efficacy if it is applied at the early stages of the disease.

Genotyping is a highly accessible and potentially informative method for identifying cases or outbreaks of $K$. pneumoniae and tracking the source of spreading infections. MLST is considered a particularly good method for typing bacterial pathogens, and it is used to characterize the genetic relationships between bacterial isolates [11]. Several studies on $K$. pneumoniae infections in humans and other animals have been conducted. On the other hand, little is known regarding the genotyping of $K$. pneumoniae in cattle. A previous study reported that ST116 could cause bovine mastitis [12]. More recently, seven STs were found in the $33 \mathrm{~K}$. pneumoniae strains isolated from bovine nasal swabs: ST294, ST328, ST218, ST2844, ST2541, and ST2581. In the current cases, MLST identified different isolates in calf A (ST14) and calf B (ST65), which were the first isolation of ST14 and ST65 in cattle. Several studies have isolated ST14 and ST65 from humans, and there are relationships between the $\mathrm{K} 2$ capsular serotype and pneumonia, urinary tract infection, and liver abscesses $[13,14]$. On the other hand, there is little information on ST14- and ST65-related infections in cattle, even though $K$. pneumoniae infections, as a secondary infection, are often found in several cases of aborted or premature calves, regardless of the primary cause. Because cattle are one of the main food-producing animals farmed, reducing the infection rates through a better understanding of the prevalence of $K$. pneumoniae and the genotype characterization of $K$. pneumoniae in bovine hosts can be expected to yield substantial economic benefits.

In conclusion, this is the first report of $K$. pneumoniae 
meningoencephalitis in calves. Bacterial polyserositis, including meningoencephalitis, may have occurred secondary to a systemic BVDV infection. A vaccine that accurately reflects the relevant antigenic subtypes will facilitate the prevention of BVDV in cattle. Moreover, K. pneumonia has zoonotic potential. Thus, further studies will be needed to accumulate knowledge of the associated virulence factors and pathogenesis.

\section{Acknowledgments}

This work was supported by the Animal and Plant Quarantine Agency, Korea (grant number B-1543069-2019-21-02).

\section{References}

1. Han DG, Ryu JH, Park J, Choi KS. Identification of a new bovine viral diarrhea virus subtype in the Republic of Korea. BMC Vet Res 2018;14:233.

2. Pinior B, Firth CL, Richter V, Lebl K, Trauffler M, Dzieciol M, Hutter SE, Burgstaller J, Obritzhauser W, Winter P, Käsbohrer A. A systematic review of financial and economic assessments of bovine viral diarrhea virus (BVDV) prevention and mitigation activities worldwide. Prev Vet Med 2017; 137:77-92.

3. Brock KV, Grooms DL, Givens M. Reproductive disease and persistent infection. In: Goyal SM, Ridpath J (eds.). Bovine Viral Diarrhea Virus Diagnosis, Management and Control. pp. 147-151, Blackwell, Ames, 2008.

4. Kasuya K, Takayama K, Bito M, Shimokubo N, Kawashima $\mathrm{R}$, Shibahara T. Septicemic invasive Klebsiella pneumoniae infection in a cynomolgus monkey (Macaca fascicularis) with severe diffused suppurative meningoencephalitis. J Vet Med Sci 2017;79:1167-1171.

5. Fecteau G, Smith BP, George LW. Septicemia and meningitis in the newborn calf. Vet Clin North Am Food Anim Pract 2009;25:195-208
6. Park JS, Moon HJ, Lee BC, Hwang WS, Yoo HS, Kim DY, Park BK. Comparative analysis on the $5^{\prime}$-untranslated region of bovine viral diarrhea virus isolated in Korea. Res Vet Sci 2004;76:157-163.

7. Diancourt L, Passet V, Verhoef J, Grimont PA, Brisse S. Multilocus sequence typing of Klebsiella pneumoniae nosocomial isolates. J Clin Microbiol 2005;43:4178-4182.

8. Oem JK, Hyun BH, Cha SH, Lee KK, Kim SH, Kim HR, Park CK, Joo YS. Phylogenetic analysis and characterization of Korean bovine viral diarrhea viruses. Vet Microbiol 2009; 139:356-360.

9. Katsande S, Matope G, Ndengu M, Pfukenyi DM. Prevalence of mastitis in dairy cows from smallholder farms in Zimbabwe. Onderstepoort J Vet Res 2013;80:523.

10. Munoz MA, Welcome FL, Schukken YH, Zadoks RN. Molecular epidemiology of two Klebsiella pneumoniae mastitis outbreaks on a dairy farm in New York State. J Clin Microbiol 2007;45:3964-3971.

11. Maiden MC, Bygraves JA, Feil E, Morelli G, Russell JE, Urwin R, Zhang Q, Zhou J, Zurth K, Caugant DA, Feavers IM, Achtman M, Spratt BG. Multilocus sequence typing: a portable approach to the identification of clones within populations of pathogenic microorganisms. Proc Natl Acad Sci U S A 1998;95:3140-3145.

12. Paulin-Curlee GG, Singer RS, Sreevatsan S, Isaacson R, Reneau J, Foster D, Bey R. Genetic diversity of mastitisassociated Klebsiella pneumoniae in dairy cows. J Dairy Sci 2007;90:3681-3689.

13. Shi Q, Lan P, Huang D, Hua X, Jiang Y, Zhou J, Yu Y. Diversity of virulence level phenotype of hypervirulent Klebsiella pneumoniae from different sequence type lineage. BMC Microbiol 2018;18:94.

14. Mei YF, Liu PP, Wan LG, Liu Y, Wang LH, Wei DD, Deng $\mathrm{Q}$, Cao XW. Virulence and genomic feature of a virulent Klebsiella pneumoniae sequence Type 14 strain of serotype K2 harboring $b l a_{\mathrm{NDM}-5}$ in China. Front Microbiol 2017;8:335. 